\title{
La implicación de las profesoras en la gestión universitaria
}

\author{
Marina Tomás ${ }^{1}$ \\ María del Mar Durán \\ Cristina Guillamón \\ Universitat Autónoma de Barcelona
}

\section{Resumen}

En este artículo se exponen los principales resultados obtenidos al investigar cómo ejercen la participación las mujeres en los órganos de gobierno de la universidad.

Partiendo de la aplicación de dos estrategias metodológicas, la observación sistemática categorial y la entrevista grupal, se concluye que existen ciertas diferencias de comportamiento entre géneros en las reuniones de toma de decisiones. Además, se constatan otros aspectos relacionados con la calidad de la decisión, con el llamado "estilo femenino" de participación y con otros factores, además del género, que parecen influir en la forma de gestionar de las profesoras universitarias.

Palabras Clave: toma de decisiones, universidad, estilos de participación, género, ejercicio del poder.

\section{Summary}

This article highlights the main results obtained from research into the manner in which women participate in the university's governing bodies. Starting from the applica- tion of two methodological strategies, systematic categoric observation and group-based interview, the article concludes that there are certain behavioural differences between genders in meetings of a decision-taking nature. Furthermore, other aspects are emphasised, relating to decision quality, to the socalled "feminine mode" of participation as well as to other factors -in addition to gender- which appear to influence women university teachers' style of management.

KEYWORDS: decision-taking, university, mode of participation, gender, power.

\section{Introducción}

Las cifras demuestran que la presencia de las profesoras universitarias en los cargos de gestión universitaria es más bien escasa. Preocupadas por ello, el equipo de investigación se planteó indagar los posibles motivos de esta realidad dando respuesta a varias preguntas: ¿qué tipo de interacciones y dinámicas se dan dentro de los órganos de gobierno de la universidad?, ¿cómo actúan las profesoras dentro de dichos órganos?, ¿el comportamiento de las profesoras difiere del de sus compañeros?, ¿están satisfechas las 
profesoras sobre su participación en la toma de decisiones?, etc.

Para dar respuesta a dichas cuestiones se realizaron ocho observaciones sistemáticas categoriales en varias reuniones de órganos de gestión de diferente tipología y universidades y se llevaron a cabo dos grupos de discusión con hombres y mujeres que han tenido o tienen algún cargo de gestión en la actualidad.

Se presentan en este artículo los instrumentos utilizados, así como los resultados obtenidos, pudiéndose concluir que se aprecian ciertas diferencias en el comportamiento de profesoras y profesores cuando participan en los órganos de gobierno de las universidades, comportamientos que están influidos por varias variables como, por ejemplo, el tamaño del grupo o el sexo de la persona que dirige la reunión.

\section{La mujer en los cargos de gestión univer- sitarios en cifras}

Las mujeres han recorrido un camino lleno de dificultades e impedimentos que las han alejado de la educación y la cultura a lo largo de la historia. La incorporación de la mujer española en la universidad como estudiante no comenzó, al igual que en otros países europeos, hasta la segunda mitad del siglo XIX, y, de la misma forma que en los otros países, fue un proceso lento, teniendo que luchar, presionar e incluso engañar para demostrar una capacidad que se les negaba, como fue el caso de Concepción Arenal.

Actualmente, a inicios de siglo XXI, las cifras demuestran que las estudiantes son mayoría en las aulas. Las mujeres representan, según datos del curso 2005-06 el 54,09\% del estudiantado universitario en España y obtienen el 60,65\% de los títulos académicos (INE, 2007).

En segundo lugar, se debe estudiar la incorporación de la mujer como personal do- cente e investigador. En este sentido, para el mismo curso, las profesoras representaban un $35,14 \%$, siendo el porcentaje menor a medida que se asciende de categoría profesional, tal como han estudiado Izquierdo et al. (2004) o como se mencionó en el Seminario interuniversitario sobre Políticas universitarias de género celebrado en la UAB en junio de 2006.

Finalmente, cabe hablar de la mujer en relación a los cargos de gestión. En este sentido, se puede afirmar que las diferencias respecto a sus compañeros aún son más notorias. Tanto es así que durante el curso 2005-06 sólo 4 de las 73 universidades del territorio español estaban dirigidas por una Rectora (Universidades de Girona, Málaga, Oberta de Catalunya y Ramon Llull), una cifra poco satisfactoria si tomamos en consideración que hace 25 años que Elisa Pérez Vera fue nombrada Rectora, convirtiéndose en la primera Rectora española.

En la tabla n. 1 se presenta el número de profesoras y profesores que ocupaban ciertos cargos de gestión (rectorado, vicerrectorado, decanato, dirección de escuela universitaria y de departamento) en las Universidades Autónoma de Barcelona, de Barcelona, Pompeu Fabra y Ramon Llull para el curso 2005-06.

Los datos que se extraen de esta tabla son muy ilustrativos. Tan sólo, como ya se indicó, una de las cuatro universidades está presidida por una Rectora, quien ha decidido formar un equipo rectoral paritario. También se puede extraer de las cifras que los rectores suelen conformar equipos eminentemente masculinos (porcentaje de vicerrectores próximo al 80\%), exceptuando la UAB, que ha formado un equipo de gobierno paritario.

Si se toman en consideración los decanatos, se puede afirmar que este cargo está altamente masculinizado en todas las universidades que formaron parte del estudio y lo mismo se puede decir de la dirección de las escuelas universitarias, a excepción de la 


\begin{tabular}{|c|c|c|c|c|c|c|c|c|}
\hline \multirow{2}{*}{$\begin{array}{l}\text { Universidad } \\
\text { Cargo }\end{array}$} & \multicolumn{2}{|c|}{ UAB } & \multicolumn{2}{|c|}{ UB } & \multicolumn{2}{|c|}{ UPF } & \multicolumn{2}{|c|}{ URL } \\
\hline & M & H & $\mathbf{M}$ & H & M & H & M & H \\
\hline Rector/a & 0 & 1 & 0 & 1 & 0 & 1 & 1 & 0 \\
\hline$\%$ & 0 & 100 & 0 & 100 & 0 & 100 & 100 & 0 \\
\hline Vicerrector/a & 6 & 4 & 2 & 7 & 2 & 8 & 1 & 2 \\
\hline$\%$ & 60 & 40 & 22,22 & 77,78 & 20 & 80 & 25 & 75 \\
\hline Decano/a & 3 & 8 & 5 & 13 & 1 & 8 & 0 & 8 \\
\hline$\%$ & 27,27 & 72,73 & 27,77 & 72,73 & 11,11 & 88,89 & 0 & 100 \\
\hline Director/a Escuela & 1 & 2 & 2 & 0 & 1 & 3 & 3 & 7 \\
\hline$\%$ & 33,33 & 66,67 & 100 & 0 & 25 & 75 & 30 & 70 \\
\hline Director/a Departamento & 12 & 42 & 26 & 77 & 1 & 8 & & tos) \\
\hline$\%$ & 22,22 & 77,78 & 25,24 & 74,76 & 11,11 & 88,89 & & \\
\hline
\end{tabular}

UB, donde las dos escuelas están dirigidas por una directora.

Finalmente, se ha estudiado el sexo de la persona que ocupa la dirección de los departamentos. En este caso también se observa una predominancia del sexo masculino, siendo la UB la universidad con más directoras de departamento, seguida de la UAB y la UPF.

Para concluir este apartado, mencionar que las cifras recogidas demuestran que a pesar que cada vez más mujeres acceden a la universidad, éstas representan una minoría dentro del colectivo de personal docente e investigador (sobre todo en los niveles más altos de la carrera académica) y aún están menos presentes en los cargos de gestión de la universidad, que continúan siendo ejercidos en gran mayoría por profesores.

\section{Metodología}

En vistas a la necesidad de indagar en los motivos de la falta de presencia femenina en las instancias de poder en las universidades, el grupo de investigación se planteó una serie de objetivos.
1. Describir y comprender el tipo de interacción y las dinámicas en los órganos de gobierno de las universidades.

2. Describir el comportamiento de las profesoras en función del tipo de interacciones.

3. Captar el grado de satisfacción de las profesoras con relación a su participación en la toma de decisiones en los órganos de gobierno colegiados.

4. Estudiar las dinámicas existentes en los órganos de gobierno con relación a la posible influencia que ejerza el género.

La fundamentación teórica de la investigación se basa en estudios sobre pautas de interacción en los grupos y sobre el género en relación con dichas pautas de interacción. Se escogieron tres aportaciones que parecieron relevantes y de interés para guiar el proceso.

Siguiendo un orden cronológico, la primera de ellas es la realizada por Robert F. Bales en los años cincuenta (Vendrell, 1999). Es el autor más emblemático de la llamada orientación interaccional, puesto que ideó un sistema de categorías para el análisis del 
proceso de interacción en el pequeño grupo. Para los autores de esta orientación, los grupos son sistemas de individuos que interactúan y la interacción se considera como una secuencia de actos comunicativos. Así, cada acto de un miembro del grupo puede ser comprendido por los demás y traducido a una frase sencilla, siendo el acto de tipo verbal o no verbal.

Bales configuró un sistema de doce categorías que permitía clasificar el tipo de actos emitidos por cada miembro. Cada categoría comprendía una serie de conductas observables: seis relacionadas con la parte instrumental del grupo (aquello que el grupo hace) y seis relacionadas con la parte socioafectiva (la relación interpersonal que acompaña a aquello que el grupo hace). La teoría de Bales considera que cada acto que se da en un grupo tiene cabida en una de las doce categorías. A través de la observación sistemática se pueden identificar las distintas categorías que se dan en el proceso de interacción de un pequeño grupo y, una vez identificadas éstas, poseemos un mapa de la dinámica del proceso de interacción que nos ayuda a comprender fenómenos y a analizar el proceso grupal.

La segunda aportación teórica ha sido la de Maria Lluisa Fabra. La autora estableció una serie de modificaciones en las categorías propuestas por Bales a fin de adaptar el modelo a su objeto de estudio: detectar la incidencia del género en la interacción en las aulas de educación secundaria (Moreno y otros, 1992).

Por proximidad teórica y concordancia con los objetivos de investigación, ha sido la aportación de Fabra la que más ha influido en nuestro diseño, llegando la propia autora a colaborar en el mismo. Así, se parte de una definición de roles y scripts, a modo de categorías de observación, propuestos por la autora, que se adaptaron al objeto de estudio y que fueron modificados después de una prueba piloto.
Finalmente, cabe mencionar el estudio de Hawkins y Power (1999), en el cual, partiendo también de una adaptación de las categorías de Bales, se analizaba la interacción en 18 grupos pequeños de toma de decisiones a fin de descubrir si existían diferencias de género en cuanto al número y al contenido de las preguntas realizadas en el seno del grupo.

En el estudio realizado se definieron en un principio 10 categorías, que finalmente fueron reducidas a 8, de las cuales seis se consideran roles y cuatro, scripts. Se entiende por rol de equipo, el tipo de intervención que emite cada persona del grupo a lo largo de una reunión y por scripts, lo que serían las formas de responder al ejercicio de la influencia o de ejercer dicha influencia, manifestadas, sobre todo, en la comunicación no verbal. Así, a una misma interacción le corresponderían dos categorías: un rol y un script.

A continuación se sintetiza la descripción de cada una de las categorías definidas. Los seis roles iniciales fueron:

- Rol iniciador: persona que plantea un nuevo tema, una cuestión o manifiesta una opinión que es comentada por el resto del grupo.

- Rol co-iniciador: persona que añade algún aspecto relevante al comentario de la iniciadora o que introduce alguna variación respecto al tema propuesto por ésta. - Rol opositor: persona que muestra intención de dar un giro a la discusión, por discrepancias relacionadas con el contenido o por la no aceptación de quien ejerce el liderazgo en ese momento.

- Rol seguidor: persona que asiente ante las aportaciones de las demás sin añadir argumentos "de propia cosecha". Repite lo dicho por otros en sus intervenciones.

Los cuatro scripts a observar fueron:

- Script asertivo: persona que utiliza un tono razonable, decidido y amable.

- Script no asertivo: persona que duda cuando interviene, habla en voz baja y uti- 
liza preferentemente el condicional y no el presente ("yo diría", "si quisieran"... etc. - Script agresivo: persona que habla desde una postura de superioridad o con desprecio hacia los demás. Interrumpe, desvaloriza, etc.

- Script "de huida": persona que sale de la sala a media discusión, o bien habla con la persona que tiene al lado, etc.

Una vez definidas las categorías teóricas, se estaba en disposición de iniciar el estudio de campo. La metodología adecuada a los objetivos relativos a describir y comprender los tipos de interacciones en los órganos de gobierno y las conductas asociadas a éstos, no podía ser otra que la observación y partiendo de la definición teórica de categorías, el sistema de observación más adecuado era, sin duda, el sistemático-categorial.

Para la recogida de datos en el campo, elaboramos una pauta de observación "a medida" de nuestro objeto de estudio, que modificamos una vez llevada a cabo la prueba piloto. Reproducimos en la tabla n. 2 la versión definitiva de dicha pauta.

Para intentar alcanzar el objetivo de captar el grado de satisfacción de las profesoras participantes en los órganos de gobierno, pareció que los grupos de discusión eran la metodología que mejores resultados ofrecería.
Autores como Krueger (1991) y Morgan (1991) ponen de manifiesto que los grupos de discusión o "focus groups" son una herramienta que permite recoger los diferentes tópicos en torno a un tema de discusión, estudiar percepciones, sentimientos y actitudes a los que sería difícil acceder mediante otras metodologías, puesto que la situación grupal favorece las confesiones individuales y permite conocer los tópicos más ampliamente compartidos entre los miembros del grupo.

Las cuestiones que orientaron la facilitación de los grupos de discusión por parte de la persona moderadora fueron las siguientes:

- Diferencias en las intervenciones (contenido) que se hacen en las reuniones en las que se toman decisiones, en función del género.

- Diferencias en el comportamiento (forma de comunicarse) en las reuniones (grado de asertividad, etc.).

- Decisión de calidad. ¿Cuál es vuestra percepción sobre la calidad en la toma de decisiones?

- Grado de satisfacción en la participación. ¿Quién queda más satisfecho de la participación en las reuniones y de los resultados obtenidos en las decisiones? Sensación subjetiva de haber conseguido los objetivos propios y los del grupo.

\section{TABLA 2. PAUTA DE OBSERVACIÓN DE ÓRGANOS DE GOBIERNO DE LA UNIVERSIDAD}

\begin{tabular}{|c|c|c|c|}
\hline Fecha de la observación: & Órgano observado: & Persona c & observadora: \\
\hline Núm. personas que forman la reunión & Hombres: & Mujeres: & Total: \\
\hline Núm. personas que han participado & Hombres: & Mujeres: & Total núm. Participantes: \\
\hline Persona iniciadora & Persona Co-iniciadora & Persona Opositora & Persona seguidora \\
\hline Asertiva & & & \\
\hline No-asertiva & & & \\
\hline Agresiva & & & \\
\hline De huida & & & \\
\hline
\end{tabular}

Nota: Hay que introducir las intervenciones para cada tema diferente, por lo tanto pueden haber más intervenciones que personas componen la reunión. 


\section{Análisis e interpretación de los resultados}

En este apartado se hace referencia a los resultados obtenidos a través de las observaciones y las entrevistas grupales o focus group realizados.

\subsection{Las observaciones}

El grupo de investigación ha realizado un total de ocho observaciones a diferentes órganos de gobierno de dos universidades, la Universidad Autónoma de Barcelona (UAB) y la Universidad de Barcelona (UB).

Los órganos de gobierno observados han sido: Comisión de investigación, Consejos de departamento (de diferentes áreas de conocimiento), Junta permanente y Comisión académica. Tres de ellas fueron dirigidas por mujeres y cinco por hombres. Mientras que las profesoras han dirigido un grupo pequeño, uno mediano y uno grande, en el caso de sus compañeros, cuatro han dirigido un grupo de tamaño mediano y uno, un grupo de tamaño grande.

En el total de las observaciones que se han realizado han participado 199 personas, de las cuales 97 mujeres y 102 hombres.

Respeto al número de intervenciones clasificadas en scripts, no se aprecian diferen-

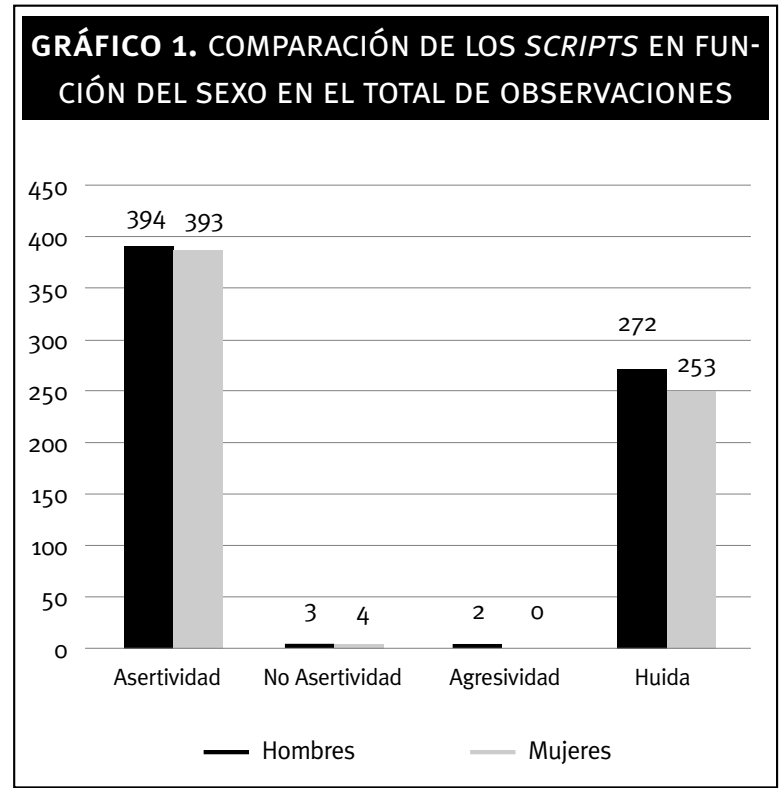

cias respecto al sexo. Sólo en el caso de intervenciones con script agresivo, que las ejercen los varones (gráfico n. 1).

Con respecto a los roles (gráfico n. 2) se observa una tendencia a que el sexo masculino sea más iniciador y juegue un rol de opositor con mayor frecuencia. Por otra parte, quedan más representados por el sexo femenino los roles de coiniciador y seguidor.

\subsection{Los grupos de discusión o focus group}

Los grupos de discusión celebrados fueron dos. En el primero participaron siete personas, cinco mujeres y dos hombres. La diferencia en el número entre unas y otros fue debida a la no asistencia de algunos de los hombres convocados. En el segundo grupo de discusión los participantes fueron cinco personas: cuatro señoras y un señor. Mientras que sólo una profesora faltó a la cita, fueron siete profesores convocados los que no acudieron, habiéndose comprometido a hacerlo.

Una de las primeras cuestiones que inevitablemente ocupan el debate dentro de los focus group respecto las relaciones entre órganos de gobierno en la universidad y género es la falta de presencia de mujeres en los cargos de gestión, especialmente en los de más alto nivel.
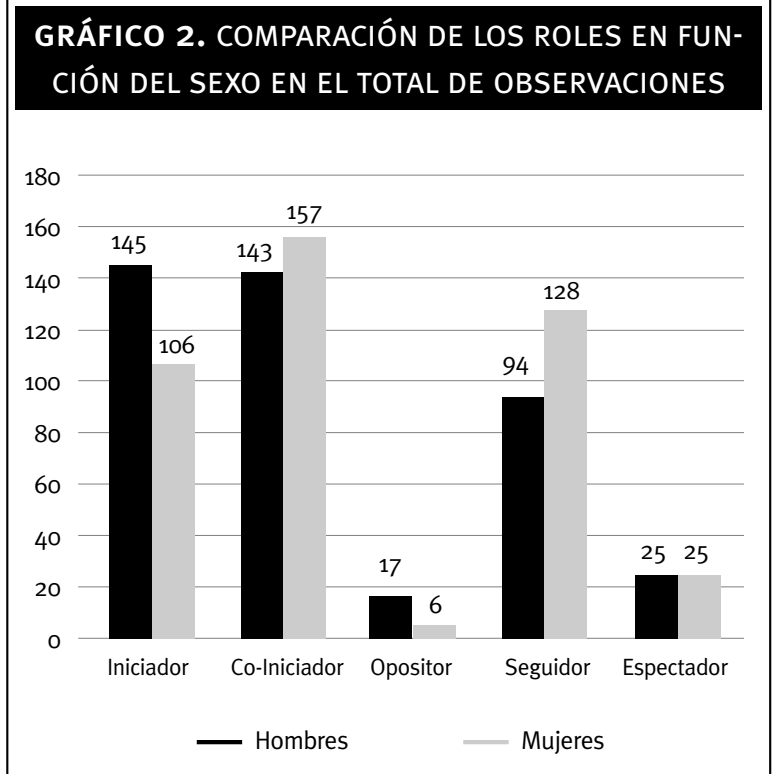
Los participantes constatan que, aparte de la creciente incorporación de la mujer a la educación universitaria e, incluso, la feminización de determinadas áreas de conocimiento, la representación femenina en los órganos de gobierno universitarios sigue siendo sensiblemente inferior en comparación con la población masculina. En la universidad, como afirmaba una de las participantes, "los cargos de poder están en manos de los hombres (...) y esto si que es un desequilibrio, independientemente de que haya hombres con un estilo femenino".

Y las mujeres que finalmente acceden, suelen reunir una serie de características sociodemográficas determinadas como tener una edad avanzada: "En los equipos de gobierno las mujeres que hay no son extremadamente jóvenes, son mujeres mayores, con hijos ya grandes, con una educación diferente. (...) Las mujeres tienen una media de edad más alta que los hombres".

Se constata, pues, como punto de partida que la gestión en la universidad sigue siendo un asunto mayoritariamente masculino, y que "sin un impulso parece que esto no deba cambiar".

La presencia de las mujeres en los órganos de gestión se justifica, en cualquier caso, tanto por cuestiones de justicia e igualdad social como por motivos de eficiencia y calidad en la toma de decisiones, como se argumentará más adelante. De hecho, una de las premisas de la investigación, y en torno la cual los participantes de los focus group se ubicaron en términos mayoritariamente favorables, es que el género introduce diferentes sensibilidades en la toma de decisiones, las cuales deben estar presentes para garantizar la calidad en el proceso y en los resultados. Así lo explicaba la coordinadora del grupo de investigación:

"Hay diferentes sensibilidades en la toma de decisiones de calidad. Hay diferentes maneras de ver la gestión de la universidad, la manera de funcionar de la uni- versidad... Hay diferentes sensibilidades entre los hombres y las mujeres. Ésta es la hipótesis de la cual partimos".

El género no puede convertirse en una categoría absoluta para la explicación de todas las diferencias en las maneras y estilos de participación. Pese a que la influencia del género es incuestionable, esta no agota las posibles explicaciones a la variabilidad entre individuos ni ejerce un efecto homogeneizador en las conductas de grupo.

Teniendo en cuenta la importancia que en su definición tienen los factores contextuales y de grupo, los argumentos debatidos en los focus group establecen una caracterización de lo que podríamos denominar una "forma femenina" de participación en la toma de decisiones que se sintetizan a continuación.

En primer lugar, por exhibir un estilo directo y conciso en cuanto a la participación, es decir, las mujeres tienden a realizar menos intervenciones públicas, y éstas, cuanto se realizan, suelen tener un carácter más concreto y resolutivo, en el sentido que suelen estar más orientadas a la resolución del problema que se discute y estar fundamentadas en razonamientos consistentes: "los hombres tienden a hablar y a echar un discurso, sentar los principios..., y el resto a escuchar. Las mujeres, todo lo contrario, no hablamos. En ese ambiente las mujeres no participan. Hacemos intervenciones más concretas, más centradas, menos largas".

"Otro elemento es el sentirse representante de opiniones. Esto es más masculino que femenino. Cuando intervengo, intervengo representando, mientras que en general el estilo femenino es más de decir: desde mi opinión, perspectiva..."

De acuerdo con el sentir de una participante, este atributo femenino guarda relación con la tendencia de las mujeres a apoyarse en terceras personas como expresión de una acti- 
tud más democrática y como una maniobra indirecta para la consecución de las propias finalidades, producto de un aprendizaje social en virtud del cual "una mujer no se considera autorizada a expresar deseos en público y a hacer por conseguirlos".

Otra característica de este estilo femenino de participación se relaciona con el mayor pragmatismo que las mujeres suelen mostrar en sus intervenciones.

Forma parte de esta sensibilidad femenina una atención a los aspectos relativos al proceso y a ocupar, como decía uno de los participantes, "el espacio fronterizo". Esta atención al proceso se relaciona, por un lado, con una preocupación por el desarrollo de la reunión y de la dinámica del grupo, aunque también tiene relación con una concepción más amplia sobre el trabajo en grupo y los elementos que pueden optimizar su funcionamiento.

Las causas que explican las diferencias se podrían agrupar según se ponga el énfasis en la participación e implicación de las mujeres en los órganos de gobierno, en el interés por acceder a los órganos de gobierno, o las atribuibles al grado de satisfacción en la toma de decisiones en éstos.

Las diversas causas que pueden contribuir a explicar las diferencias en la participación de las mujeres en los órganos de gobierno, según se desprende de las entrevistas grupales, pueden ser:

1. Tipología de contexto: reuniones a nivel de departamento, facultad, rectorado...

2. Composición del grupo: tamaño, género...

3. Características personales: categoría profesional, estatus, estado civil...

Otro de los tópicos que se pretendía que abordaran los participantes de los focus group era la posible influencia del género en la calidad de las decisiones que se toman.
La calidad de una decisión tiene relación con el grado en que esta contribuye a la consecución de los objetivos del grupo (un órgano de gobierno, una comisión, o la institución en su conjunto).

Atendiendo a factores de proceso, la calidad de una decisión también tiene relación con la calidad del proceso mismo de participación, si el sistema es democrático, a través del cual se establece la toma de decisiones. En este sentido se diferencian dos condiciones que el proceso de toma de decisiones debe tener en cuenta para garantizar la calidad: por un lado, debe asegurarse que en el grupo encargado de la toma de decisiones están presentes los diferentes intereses y sensibilidades que se encuentran en la comunidad a la cual representa. El género, en la medida que pueda asociarse como constitutivo de una sensibilidad o manera de hacer diferenciada, debe encontrar una representación adecuada en el foro en el que se tomen decisiones para favorecer que estas representen a la comunidad universitaria en su diversidad.

Se debe garantizar que durante el proceso de toma de decisiones se reúnan aquellas condiciones que permitan que todas las visiones representadas puedan participar y sean igualmente tenidas en cuenta.

La cuestión de la representatividad introduce entre los participantes preguntas sobre la eficacia y el interés de las políticas de paridad en los órganos de gobierno. Incluso, partiendo de la premisa que adelantaban algunos participantes que "si hay mujeres, seguro que es mejor porque las decisiones tienen más información" y que "mitad y mitad enriquece el grupo", parece ser que existe un cierto consenso en que la paridad, por sí misma, no garantiza la calidad.

\section{Conclusiones}

Por último se sintetizan las principales conclusiones obtenidas en el estudio. 
En relación con la gestión universitaria en general:

- La incorporación de la mujer a la gestión universitaria debe justificarse tanto por razones de justicia e igualdad social como por motivos de eficiencia y calidad en la toma de decisiones.

- La universidad no es pionera en la asunción y representación de cargos de gestión por parte de las mujeres. Pese a la creciente presencia de la mujer en los órganos de gobierno universitarios, la visualización de la mujer sigue siendo menor que la del hombre. En el sector productivo, la representación de mujeres tiende a ser mayor que en el ámbito universitario.

- Los motivos que pueden explicar esta menor presencia de la mujer en la gestión universitaria son múltiples y se relacionan tanto con factores estructurales como culturales. La categoría profesional y la edad, la experiencia en gestión, las responsabilidades familiares o atención a terceras personas son algunas de las causas que explican la menor participación de las mujeres en los órganos de gobierno.

En relación con la participación en la toma de decisiones en la universidad:

- Las intervenciones de los participantes son casi siempre constructivas, positivas y en la misma dirección que marca quien coordina la reunión. Encontramos poquísimas intervenciones de las denominadas "opositoras". Y, cuando están, las interpretan los hombres. Esto puede tener varias explicaciones: o bien que no hay miembros en los órganos que representen posiciones contrarias a las marcadas por los equipos de gobierno territoriales y centrales, que no consideran que valga la pena intervenir oponiéndose o que las propuestas de los responsables de estos órganos son tan acertadas y están tan bien preparadas que no dan lugar a la crítica. - El tono de las intervenciones es poquí- simas veces agresivo y en las pocas ocasiones en que así se puede considerar se trata de intervenciones hechas por hombres.

- Aunque la influencia del género en las formas y estilos de participación resulta incuestionable, éste no puede convertirse en la única explicación de las diferencias de comportamiento perceptibles en los procesos de toma de decisiones.

- Es posible identificar factores que modulan la forma en que el género se manifiesta. Así, la tipología y composición del grupo, la experiencia profesional, la categoría profesional de los participantes, etc. influyen en la manera de tomar decisiones. La cantidad de personas que componen una reunión parece ser una variable muy influyente en la calidad de la participación, pudiendo decir que cuando el grupo es pequeño o mediano existe más participación, proporcionalmente hablando, que cuando el tamaño es muy grande.

- El estilo de liderazgo de la persona que coordina es otro factor que parece condicionar el tipo de intervención. De las ocho reuniones observadas sólo tres han sido coordinadas por mujeres, lo cual quizá favorece que el rol iniciador sea más representado por los hombres que por las mujeres. Aun así, de acuerdo con las observaciones realizadas, en las reuniones coordinadas por mujeres parece que hay mayor participación de las mujeres ejerciendo el rol de coiniciadoras y seguidoras. En general podríamos decir que cuando la reunión es dirigida por una mujer hay más participación y ésta es más homogénea (entre hombres y mujeres, de todos los roles y todos los scripts...). Por lo tanto, podríamos decir que en el caso que dirigiese la reunión una mujer, aumentaría la calidad de la toma de decisiones si se entiende que ésta se ve incrementada por la mayor y mejor distribución de la participación. 
- Además del género de quien coordina una reunión, parece que influye decisivamente en la calidad de la participación y de la toma de decisiones el estilo de coordinación y/o tipo de liderazgo de quien dirige la reunión. A grandes rasgos, y con la debida cautela, es posible caracterizar un "estilo femenino" de participación. Vendría definido por el uso de intervenciones directas y concisas, la búsqueda del diálogo y el consenso, la articulación de planteamientos más pragmáticos y la preocupación por los aspectos relacionados con el proceso y no sólo con el contenido de la toma de decisiones, hecho que afecta positivamente en la participación.

En relación con la calidad en la toma de decisiones en la universidad:

- La calidad en la toma de decisiones depende tanto del grado en qué la decisión adoptada contribuye a la consecución de los objetivos del grupo, como del proceso mismo de participación a través de como se toma la decisión. Entre los factores procesuales, revisten especial importancia la representación de los diferentes intereses implicados y la participación en términos de igualdad.

- El género, como aspecto constitutivo de las relaciones interpersonales, a veces está presente de manera tácita en las dinámicas que se generan en el interior de los órganos de gobierno, lo cual invita a reconsiderar cualquier definición de calidad en la toma de decisiones en términos puramente técnicos.

\section{Referencias bibliográficas}

Fabra, M.Ll. (1992). "La interacción en las aulas de enseñanza secundaria: incidencia del género en Ministerio de Asuntos Sociales" (1992). Del silencio a la palabra. Madrid: Instituto de la Mujer
Guillamón, C. (2006). El desenvolupament professional, l'assumpció de càrrecs i l'estil de lideratge de les directores de departament a la universitat catalana. Trabajo de doctorado inédito. Departamento de Pedagogía aplicada. Universitat Autònoma de Barcelona.

Hawkins, K. \& Power, C.B. (1999): “Gender differences in questions asked during small decisiónmaking group discussions". Small Group Research, $30(2), 235-256$.

Izquierdo, M. J. (Dir.) (2004). El sexisme a la UAB. Propostes d'actuació $i$ dades per a un diagnòstic. Bellaterra: Edicions de la UAB.

Krueger, R.A. (1991). El Grupo de discusión. Guía práctica para la investigación aplicada. Madrid: Ediciones pirámide.

Morgan, D. (1991). Focus Group as Qualitative Research. Califòrnia: Sage.

Polítiques universitàries de gènere. Seminari Interuniversitari. Juny de 2006. Disponible en: www.uab.cat/observatori-igualtat

Vendrell, E; Ayer, J.C. y Molist, E. (1999) “L'orientació de la interacció en Vendrell", E. (coord) Dinàmica de grups i psicología dels grups. Barcelona: UB.

Web del Instituto Nacional de Estadística: www.ine.es

\section{Notas}

${ }^{1}$ La presente comunicación se elabora a partir de L'Estudi de les dinàmiques en el sí dels òrgans de govern de le universitats des de la perspectiva del gènere" financiado por la Agència de Gestió d'Ajunts Universitaris i de Recerca (AGAUR), del Departament d'Innovació, Universitats i Empresa (DIUE) de la Generalitat de Catalunya (convocatoria 2005 RDG 10.003).

DiRECCIÓN DE LA AUTORA: Marina Tomàs. Universitat Autònoma de Barcelona. Facultad Ciencias de la educación (Edificio G6). Departamento de Pedagogía aplicada. 08193. Beellaterra (Cerdanyola del Vallès), Barcelona.

Correo electrónico: marina.tomas@uab.cat

Fecha de recepción del artículo: 06.II.2007

Fecha de aceptación definitiva: 16.Iv.2008 